\title{
AUTOMORPHISMS OF COMPACT RIEMANN SURFACES AND THE VANISHING OF THETA CONSTANTS
}

\author{
BY H. M. FARKAS ${ }^{1}$
}

Communicated by Maurice Heins, October 13, 1966

I. It is the purpose of this note to announce a theorem which shows that there exists a connection between automorphisms of compact Riemann surfaces and the vanishing of Riemann theta constants. In particular we shall outline the proof of the following theorem:

Theorem 1. Let $S$ be a compact Riemann surface of genus $2 g-1$, $g \geqq 2$, which permits a conformal fixed point free involution $T$. Let $\gamma_{1}, \cdots, \gamma_{2 g-1} ; \delta_{1}, \cdots, \delta_{2 g-1}$ be a canonical dissection of $S$ and let $T$ be such that $T\left(\gamma_{1}\right)$ is homologous to $\gamma_{1}, T\left(\delta_{1}\right)$ is homologous to $\delta_{1}, T\left(\gamma_{i}\right)$ is homologous to $\gamma_{g+i-1}$ and $T\left(\delta_{i}\right)$ is homologous to $\delta_{g+i-1}, i=2, \cdots, g$. Then, there exist at least $2^{g-2}\left(2^{g-1}-1\right)$ half integer theta characteristics $\epsilon_{1}, \cdots$ such that $\theta_{\epsilon_{1}}(0)=\theta_{\epsilon_{2}}(0)=\cdots=0$ and the order of the zero is $\geqq 2$.

The proof of the theorem rests on the fact that $S$ is a two sheeted nonbranched covering of a compact Riemann surface of genus $g$ and the following lemma which was proved in [1].

LemMa 1. Let $\zeta, \omega$ be equivalent special divisors of degree $G-1$ on a compact Riemann surface $S$ of genus $G$. Then if $i(\zeta \omega)=1$, where " $i$ " is the index of specialty of the divisor, there exists a half integer characteristic $\epsilon$ corresponding to the divisor $\zeta$ such that $\theta_{\epsilon}(0)=0$ and the order of the zero is $\geqq 2 . \theta$ is of course the Riemann theta of $S$.

II. Let $\hat{S}=S / T$ denote the compact Riemann surface of genus $g$ which is covered by $S$. Then all the functions and differentials which exist and are well defined on $\hat{S}$ may be lifted to $S$ and are well-defined objects thereon. As a matter of fact all such lifted functions and differentials will be invariant under the involution $T$ and conversely all objects on $S$ which are invariant under $T$ are well defined on $\hat{S}$. There are, however, objects which are not well defined on $\hat{S}$ but are well defined on $S$. For example, let $\hat{\theta}_{\alpha}$ and $\hat{\theta}_{\beta}$ be two odd Riemann thetas associated with $\hat{S}$ such that $\alpha+\beta \equiv \delta$ where $\delta$ is the characteristic $\left(0, \cdots, 0 ; \frac{1}{2}, 0 \cdots 0\right), \alpha, \beta$ half integer characteristics. Then the quotient $\hat{\theta}_{\alpha} / \hat{\theta}_{\beta}$ is not well defined on $\hat{S}$ for analytic continuation of

1 Research partially supported by NSF GP 3452 . 
$\hat{\theta}_{\alpha} / \hat{\theta}_{\beta}$ around the cycle $\delta_{1}$ on $\hat{S}$ carries $\hat{\theta}_{\alpha} / \hat{\theta}_{\beta}$ into $-\hat{\theta}_{\alpha} / \hat{\theta}_{\beta}$. Such functions will be called multiplicative functions and they will be discussed in detail by $\mathrm{H}$. E. Rauch and the author in a forthcoming paper [2]. The crucial thing here is that $\hat{\theta}_{\alpha} / \hat{\theta}_{\beta}$ is well defined on $S$ and is a single valued meromorphic function on $S$ with $2 g-2$ zeros and $2 g-2$ poles. Utilizing now the well-known result that associated with an odd theta there is an Abelian differential of first kind with double zeros, we are through. For let $d \hat{\omega}_{\beta}$ be the Abelian differential of first kind associated with $\hat{\theta}_{\beta}$ on $\hat{S}$, and let $d \omega_{\beta}$ be the lifted differential on $S$. $\left(\hat{\theta}_{\alpha} / \hat{\theta}_{\beta}\right) d \omega_{\beta}$ is then an Abelian differential of first kind on $S$ and its zeros are precisely the same as the zeros and poles of $\hat{\theta}_{\alpha} / \hat{\theta}_{\beta}$. Hence we have shown that on $S$ there exist equivalent special divisors $\zeta, \omega$ of degree $G-1=2 g-2$, the respective zero and polar divisors of $\hat{\theta}_{\alpha} / \hat{\theta}_{\beta}$, such that $i(\zeta \omega)=1$. Hence by the lemma there exists a half integer characteristic $\epsilon$ such that $\theta_{\epsilon}(0)=0$ and the order of the zero is $\geqq 2$.

It is now easy to compute a lower bound for the number of half integer characteristics $\epsilon_{i}$ for which $\theta \epsilon_{i}(0)=0$ and the order of the zero is $\geqq 2$. There are at least as many half integer characteristics as there are pairs of odd theta characteristics $\alpha, \beta$ such that $\alpha+\beta \equiv \delta$. This number is clearly equal to $2^{g-2}\left(2^{g-1}-1\right)$. The proof of Lemma $1 \mathrm{im}-$ plies that different pairs $(\alpha, \beta)$ correspond to different $\epsilon$.

Corollary 1. If the genus of $S$ is 3 then $S$ is hyperelliptic.

Proof. By Theorem 1, there exists one half integer characteristic $\epsilon$ such that $\theta_{\epsilon}(0)=0$ and the order of the zero is $\geqq 2$. However, it is known that for a compact Riemann surface of genus 3, 2 is an upper bound for the order of vanishing of a theta [3]. Hence the characteristic $\epsilon$ is an even characteristic and it is well known that a necessary and sufficient condition for a compact Riemann surface of genus 3 to be hyperelliptic is that one even theta vanish at the origin.

The result may also be obtained directly in this case by observing that the lifted function $\hat{\theta}_{\alpha} / \hat{\theta}_{\beta}$ is a function with two poles.

\section{REFERENCES}

1. H. M. Farkas, Special divisors and analytic subloci of Teichmueller space, Amer. J. Math. 88 (1966), 881-901.

2. H. E. Rauch and H. M. Farkas, Multiplicative functions on compact Riemann surfaces (in preparation). 37-61.

3. J. Lewittes, Riemann surfaces and the theta function, Acta Math. 111 (1964),

JOHNS HOPKINS UNIVERSITY AND

YESHIVA UNIVERSITY 